\title{
Comunicação
}

\section{Programa computacional para calcular a potência requerida de máquinas e implementos agrícolas ${ }^{1}$}

\author{
Pablo Pereira Corrêa Klaver², Ricardo Ferreira Garcia ${ }^{3}$, José Francisco Sá Vasconcelos Júnior², \\ Delorme Corrêa Junior ${ }^{4}$, Wellington Gonzaga Vale ${ }^{5}$
}

\section{RESUMO}

O uso de programas computacionais no setor agrícola permite atingir objetivos específicos na área. Dentre esses, um dos mais complexos é a seleção adequada de máquinas e implementos agrícolas visando à otimização de operações agrícolas, devido, principalmente, à grande variedade de equipamentos existentes no mercado e a gama de tarefas e situações de trabalho que estas são submetidas no campo. O objetivo deste trabalho foi desenvolver um programa computacional para calcular a potência requerida de máquinas e implementos agrícolas normalmente utilizados na condução de operações de campo, desde o preparo do solo até as operações de implantação de culturas. Desenvolvido em linguagem PHP, o programa computacional baseia-se na norma ASAE D497.4 - Agricultural Machinery Management Data como referência para desenvolvimento de cálculos. A partir do programa desenvolvido, tornou-se possível a execução de tarefas para cálculos de avaliação da demanda de potência de máquinas e implementos agrícolas de forma simplificada pela internet.

Palavras-chave: força requerida, mecanização agrícola, programação.

\section{ABSTRACT}

\section{Software development to calculate the power requirement of agricultural machinery and implements}

The use of software in agriculture allows the achievement of specific objectives in the sector. One of most complex objectives is to choose properly agricultural machines and implements, mainly due to the wide variety of equipment available in the market and the range of tasks and work situations that they are subjected in the field. The objective of this study was to develop a piece of software to calculate the power requirement of farm machines and implements used to carry out field operations, from soil preparation to planting. The software was developed using the PHP language and based on ASAE Standard D497.4 - Agricultural Machinery Management Data, as reference to develop calculations. The software developed allowed the performance of tasks to calculate the demanded power of agricultural machines and implements in a simplified way using the internet.

Key words: demanded force, agricultural machinery, programming.

Recebido para publicação em 22/11/2011 e aprovado em 08/07/2013.

'Extraído da Dissertação de Mestrado do primeiro autor.

${ }^{2}$ Engenheiros-Agrônomos, Mestres. Centro de Ciências e Tecnologias Agropecuárias, Universidade Estadual do Norte Fluminense, Avenida Alberto Lamego, 2000, Parque Califórnia, 28013-602, Campos dos Goytacazes, Rio de Janeiro, Brasil. pablo@uenf.br (autor para correspondência); ferrete@uenf.br.

${ }^{3}$ Engenheiro Agrícola, Doutor. Centro de Ciências e Tecnologias Agropecuárias, Universidade Estadual do Norte Fluminense, Avenida Alberto Lamego, 2000, Parque Califórnia, 28013-602, Campos dos Goytacazes, Rio de Janeiro, Brasil. garcia@uenf.br

${ }^{4}$ Engenheiro-Agrônomo. Centro de Ciências e Tecnologias Agropecuárias, Universidade Estadual do Norte Fluminense, Avenida Alberto Lamego, 2000, Parque Califórnia, 28013602, Campos dos Goytacazes, Rio de Janeiro, Brasil. delormeminas@ hotmail.com

${ }^{5}$ Engenheiro Agrícola, Doutor. Instituto de Ciências Agrárias, Universidade Federal do Mato Grosso, Avenida Alexandre Ferronato, 1200, Setor Industrial, 78557-267, Sinop, Mato Grosso, Brasil.valewg@ufmt.br

Rev. Ceres, Viçosa, v. 60, n.6, p. 890-895, nov/dez, 2013 


\section{INTRODUÇÃO}

Atualmente, há necessidade do aumento da eficiência em todos setores da economia para a manutenção da competitividade. A otimização do projeto, adequação de maquinário, as práticas de irrigação, o desenvolvimento de sistemas de informação geográfica e posicionamento global, entre outras técnicas, estão proporcionando à agricultura ganhos crescentes, permitindo aumento da produção com redução de áreas cultivadas, insumos e danos ambientais.

Nas últimas décadas, o uso da informática e de programas computacionais trouxe consigo diversos benefícios aos setores agrícola e agropecuário, tendo sido reportado por diversos autores (Meira et al., 1996; Campos, 2009).

Segundo Klaver (2006), uma das ferramentas atuais mais usadas para o desenvolvimento de programas via internet é o PHP (Hypertext Preprocessor). O fato de seu código ser executado no servidor permite que computadores com poucos recursos de processamento executemno. Esse tipo de utilização torna mais fácil o uso do programa, já que não existe instalação de arquivos, e o usuário pode ter acesso em locais diferentes, utilizando algum dispositivo de conexão sem fio.

O PHP possui diversas características para ser tão popular na internet, como sua atitude pragmática, facilidade de uso e implementação, características de segurança e compatibilidade (Neto et al., 2009).

O servidor Apache é o mais bem sucedido servidor web livre. Foi criado em 1995 por Rob McCool, então funcionário do NCSA (National Center for Supercomputing Applications). É a principal tecnologia da Apache Software Foundation e é responsável por mais de uma dezena de projetos envolvendo tecnologias de transmissão via web, processamento de dados e execução de aplicativos distribuídos (Heck, 2007).

Segundo Camargo (2005), o MySQL é um banco de dados relacional que utiliza o SQL como forma de acessar e manipular dados armazenados. O MySQL é um sistema de banco de dados com grande disponibilidade de funções e possibilidades. Ele é muito rápido, multitarefa e multiusuário. O MySQL pode ser utilizado embutido em um programa e para vários fins, como uma simples lista de compras, galeria de imagens ou grande quantidade de dados de uma rede corporativa.

Santos et al. (2006) desenvolveram uma planilha eletrônica, implementada a partir de um algoritmo desenvolvido em Visual Basic, para determinação da potência disponível na barra de tração. Adotou-se um procedimento que permite determinar a força de tração, velocidade, potência disponível na barra de tração, o consumo horário, consumo específico, a patinagem e o coeficiente de tração do trator ensaiado.
Pranav \& Pandey (2008) desenvolveram um programa que incorpora modelos teóricos e empíricos existentes. $\mathrm{O}$ programa, escrito em Visual Basic, fornece uma coleção de painéis de entrada de dados associados com tratores, solos, pneus e implementos. O programa calcula os parâmetros de desempenho do trator, incluindo o lastro ideal em eixos traseiros e dianteiros.

Assim, o objetivo deste trabalho foi desenvolver um programa computacional, utilizando a linguagem PHP, baseado na norma ASAE D497.4 - Agricultural Machinery Management Data (ASAE, 2003), para calcular a potência requerida por máquinas e implementos agrícolas.

\section{MATERIAL E MÉTODOS}

Um programa computacional para cálculo da potência requerida de máquinas e implementos agrícolas foi desenvolvido em linguagem PHP, sendo utilizado o programa Macromedia Dreamweaver MX para edição da linguagem de marcação do hipertexto e PHP. No desenvolvimento, utilizou-se o banco de dados MySQL, que é um sistema de gerenciamento de banco de dados que utiliza a linguagem SQL - Structured Query Language - ou Linguagem de Consulta Estruturada como interface.

O programa computacional foi desenvolvido para ser executado via internet, bastando o usuário ter acesso à rede para entrar no endereço que disponibiliza o programa, sendo hospedado em um servidor Apache que se encontra em um provedor de serviços de internet nos EUA com capacidade de $300 \mathrm{MB}$.

Para a realização dos cálculos, o programa computacional baseou-se na norma ASAE D497.4 - Agricultural Machinery Management Data (ASAE, 2003), para calcular, inicialmente, a força, e, posteriormente, a potência requerida para tracionar implementos de preparo do solo - subsoladores e aplicadores de material orgânico, arados de aiveca, escarificadores, grades de discos de simples e de dupla ação tandem e offset, cortadores de palhada, cultivadores mecânicos e máquinas de semeadura.

A força exigida na barra de tração do trator, segundo a norma ASAE D497.4 (ASAE, 2003), é calculada pela equação 1 .

$\mathrm{D}=\mathrm{F}_{\mathrm{i}}\left[\mathrm{A}+\mathrm{B}(\mathrm{S})+\mathrm{C}(\mathrm{S})^{2}\right] \mathrm{W} . \mathrm{T}$

em que:

$\mathrm{D}=$ força exigida na barra de tração, $\mathrm{N}$;

$\mathrm{F}_{\mathrm{i}}=$ parâmetro adimensional de ajuste da textura do solo;

I = 1 para solo argiloso, 2 para solo médio ou 3 para solo arenoso;

A, B e C = parâmetros adimensionais específicos da máquina; 
$\mathrm{S}=$ velocidade de trabalho, $\mathrm{km} \mathrm{h}^{-1}$;

$\mathrm{W}=$ largura da máquina, $\mathrm{m}$, ou número de peças; $\mathrm{e}$

$\mathrm{T}=$ profundidade de trabalho, $\mathrm{cm}$, para implementos de preparo do solo ou 1 para outros.

De acordo com a força exigida na barra de tração, a potência exigida na barra de tração do trator pelo implemento agrícola é calculada utilizando a equação 2.

$\mathrm{P}=\mathrm{D} . \mathrm{S}$

em que;

$\mathrm{P}=$ potência exigida na barra de tração, $\mathrm{W}$;

$\mathrm{D}=$ força exigida na barra de tração, $\mathrm{N}$; e

$\mathrm{S}=$ velocidade de trabalho, $\mathrm{m} \cdot \mathrm{s}^{-1}$.

Para a determinação da potência exigida no motor do trator, utiliza-se o quadro de relação de potência para tratores agrícolas, conforme a norma ASAE D 497.4 (ASAE, 2003), em que a partir da potência nominal do motor do trator é possível conhecer a potência disponível na TDP e barra de tração. O cálculo da potência exigida no motor do trator permite recomendar o melhor trator para realizar a operação agrícola, dimensionando-se, assim, o conjunto trator e implemento mais adequado para a realização do trabalho. O fluxograma do programa desenvolvido é apresentado na Figura 1.

Para a avaliação da funcionalidade do programa computacional desenvolvido, utilizou-se a metodologia GQM Goal QuestionMetric (Gladcheff, 2001; Souza et al., 2009).

Foi disponibilizado a 31 alunos dos cursos de nível superior de Agronomia e Zootecnia um endereço eletrônico na internet permitindo o acesso livre para execução do programa. Os alunos, após fazerem uso do programa, responderam questionários de sua avaliação contendo os seguintes tópicos: funcionalidade, objetivo, projeto visual, compatibilidade, aplicabilidade, confiabilidade e usabilidade. As opções de resposta eram sim, médio ou não, com espaço para respostas adicionais quando ne-

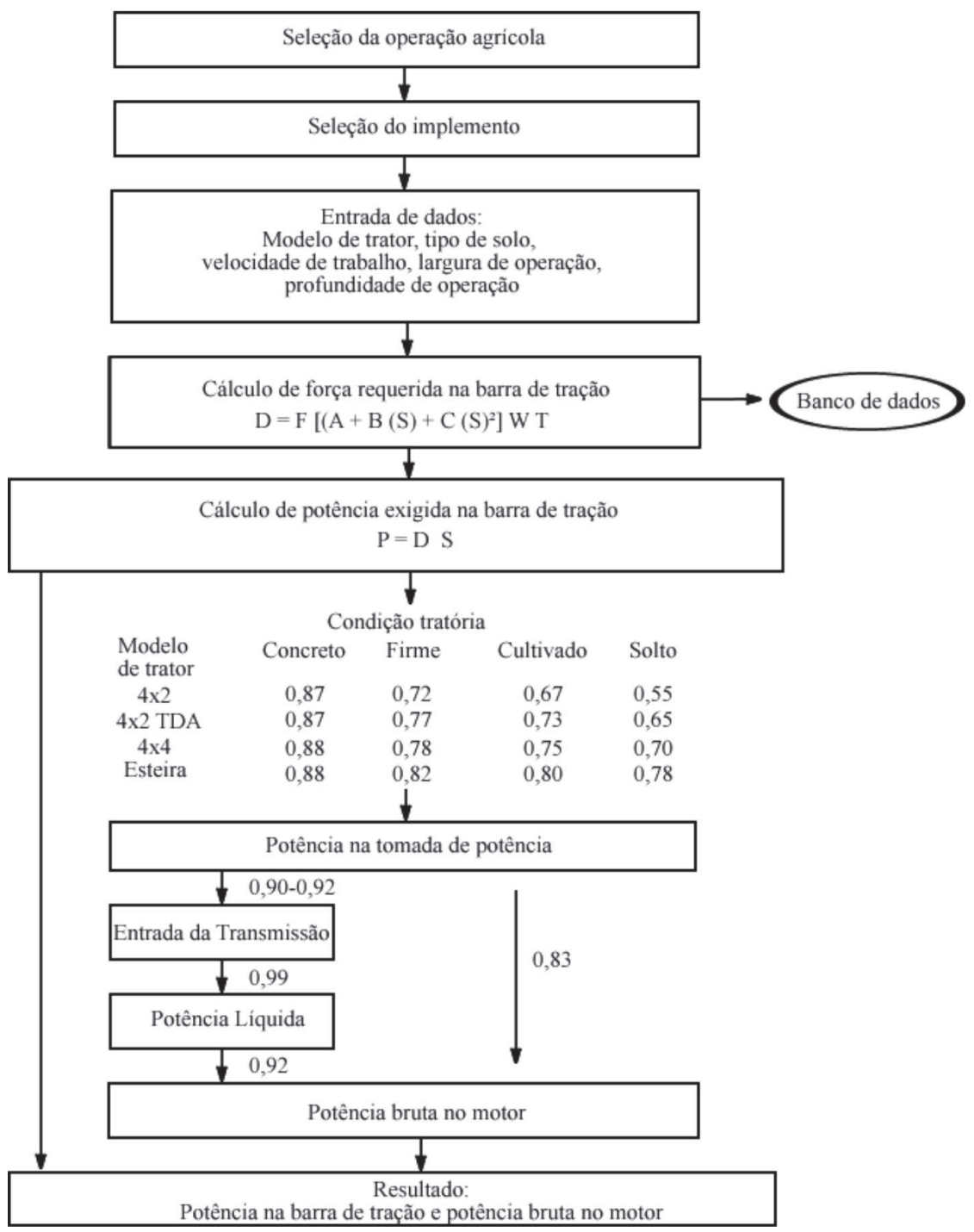

Figura 1. Fluxograma do programa desenvolvido para dimensionamento do conjunto trator/implemento.

Rev. Ceres, Viçosa, v. 60, n.6, p. 890-895, nov/dez, 2013 
cessárias. Com base nos dados coletados nos formulários respondidos, realizou-se uma avaliação estatística utilizando o teste t de Student. Todos os alunos que usaram o programa desenvolvido já haviam concluído as disciplinas de Mecânica Aplicada e Mecanização Agrícola.

Para a validação do modelo compararam-se resultados teóricos obtidos com o programa desenvolvido com dados de campo obtidos por pesquisadores realizando análise de força e potência em implementos de preparo, cultivo e semeadura equivalentes.

\section{RESULTADOS E DISCUSSÃO}

O programa computacional para avaliação da demanda de potência desenvolvido apresenta uma tela inicial onde o usuário faz a seleção da operação agrícola e do implemento utilizado (Figura 2). Em seguida, ele deve escolher o modelo de trator -4 x 2 , 4 × 2 TDA, 4 x 4 ou de esteiras, e o tipo de solo; a velocidade de operação; largura e profundidade de trabalho do implemento utilizado (Figura 3).
Após a entrada dos dados, o programa exibe o resultado da potência exigida na barra de tração e da potência bruta exigida no motor do trator (Figura 4).

A avaliação do programa computacional realizada por alunos dos cursos de Agronomia e Zootecnia está apresentada na Tabela 1 , onde se encontram as características avaliadas de funcionalidade, objetivo, projeto visual, compatibilidade, aplicabilidade, confiabilidade e usabilidade.

$\mathrm{Na}$ avaliação do programa computacional (Tabela 1) em relação a sua característica de funcionalidade, foi observado que $100 \%$ dos entrevistados aprovaram o programa. No objetivo, verificou-se que $100 \%$ dos entrevistados reconheceram o alcance do objetivo. Em relação ao projeto visual, foi observado que $92,38 \%$ dos entrevistados analisaram o visual como adequado. Já no que se refere à compatibilidade, verificou-se que $81,63 \%$ dos entrevistados não necessitaram de treinamento para utilização. Continuando a avaliação, na parte de aplicabilidade, foi constatado que $94,78 \%$ dos entrevistados acharam o

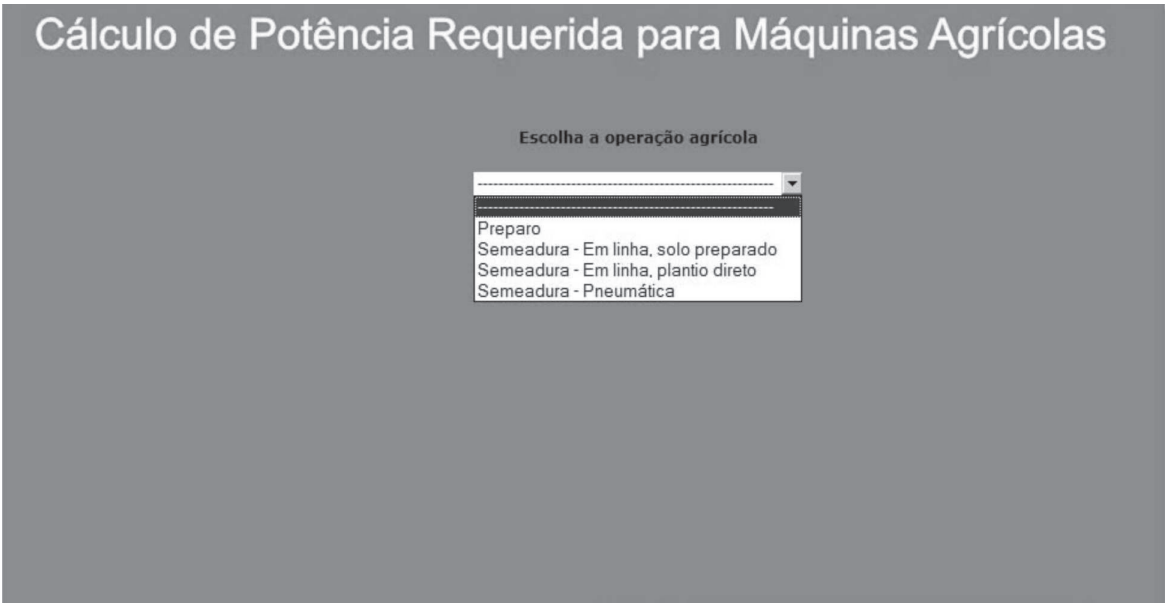

Figura 2. Tela de seleção da operação agrícola.

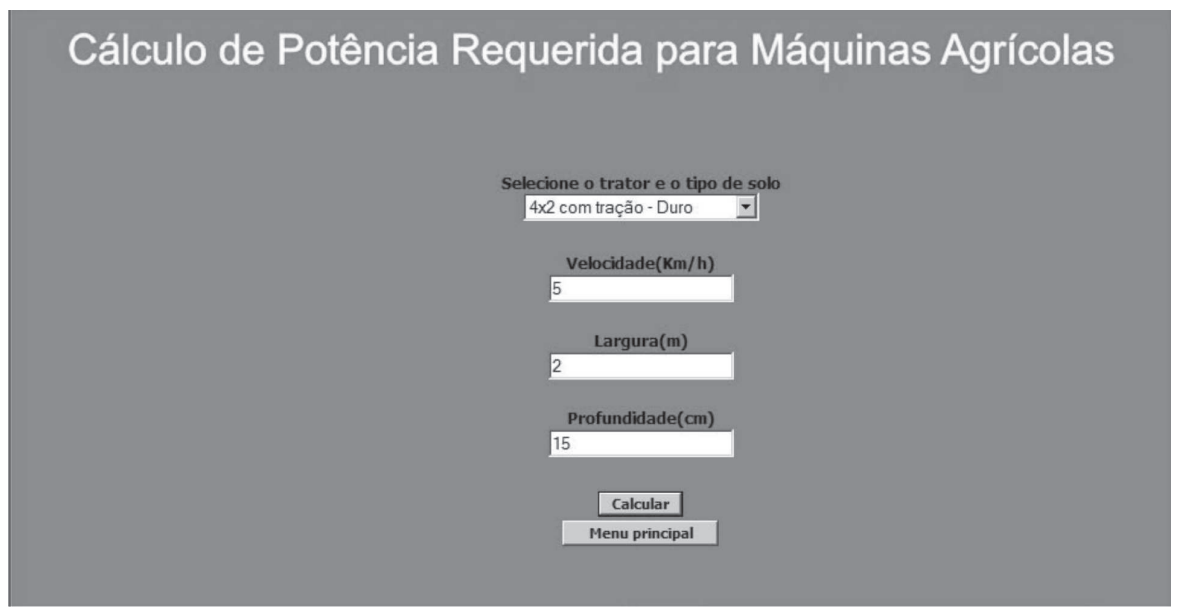

Figura 3. Tela de seleção do trator, solo, velocidade de operação, largura e profundidade de trabalho. 


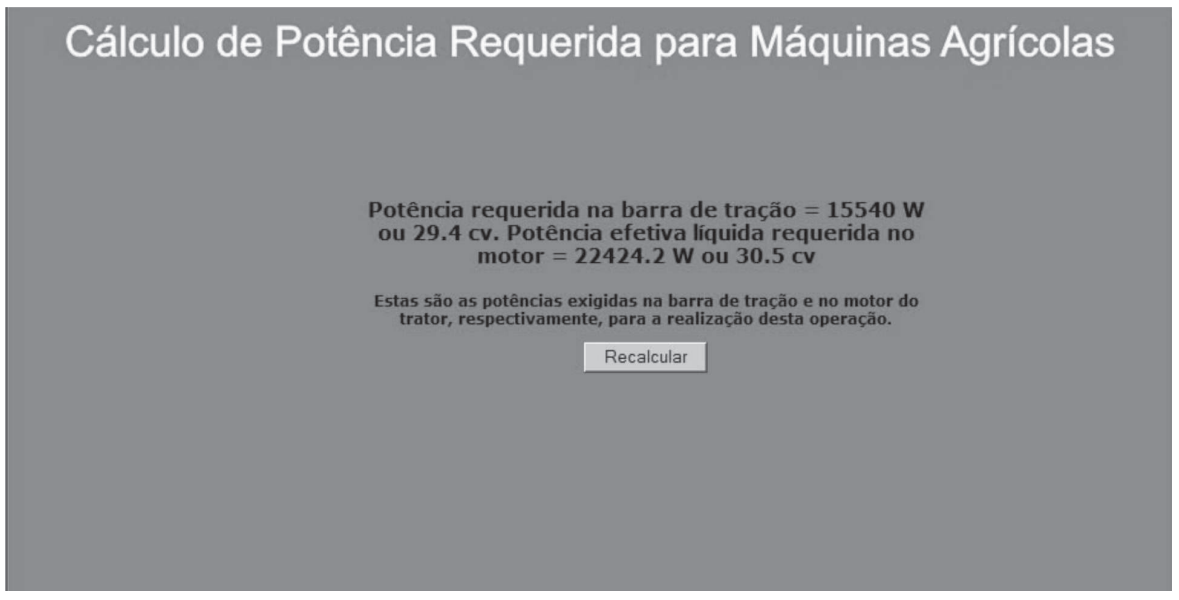

Figura 4. Valores resultantes de potência requerida na barra de tração e potência efetiva líquida requerida no motor em Watts e cavalos.

Tabela 1. Avaliação do programa computacional desenvolvido pelos alunos de Agronomia e Zootecnia da Universidade Estadual do Norte Fluminense

\begin{tabular}{|c|c|c|c|c|}
\hline Característica & & & & \\
\hline & Sim & $100,00 \%$ & $\pm 11 \%$ & $\mathrm{a}$ \\
\hline Funcionalidade & Médio & $15,24 \%$ & $\pm 9 \%$ & $\mathrm{~b}$ \\
\hline & Não & $9,54 \%$ & $\pm 6 \%$ & $\mathrm{~b}$ \\
\hline & Sim & $100,00 \%$ & $\pm 6 \%$ & $\mathrm{a}$ \\
\hline Objetivo & Médio & $9,54 \%$ & $\pm 6 \%$ & $\mathrm{~b}$ \\
\hline & Não & $9,54 \%$ & $\pm 6 \%$ & $\mathrm{~b}$ \\
\hline & Sim & $92,38 \%$ & $\pm 15 \%$ & $\mathrm{a}$ \\
\hline Projeto visual & Médio & $33,49 \%$ & $\pm 14 \%$ & $\mathrm{~b}$ \\
\hline & Não & $9,54 \%$ & $\pm 6 \%$ & $\mathrm{~b}$ \\
\hline & Sim & $37,54 \%$ & $\pm 15 \%$ & $\mathrm{a}$ \\
\hline Compatibilidade & Médio & $20,25 \%$ & $\pm 11 \%$ & $\mathrm{~b}$ \\
\hline & Não & $81,63 \%$ & $\pm 17 \%$ & $\mathrm{c}$ \\
\hline & Sim & $94,78 \%$ & $\pm 14 \%$ & $\mathrm{a}$ \\
\hline Aplicabilidade & Médio & $29,29 \%$ & $\pm 13 \%$ & $\mathrm{~b}$ \\
\hline & Não & $9,54 \%$ & $\pm 6 \%$ & $\mathrm{~b}$ \\
\hline & Sim & $100,00 \%$ & $\pm 6 \%$ & $\mathrm{a}$ \\
\hline Confiabilidade & Médio & $9,54 \%$ & $\pm 6 \%$ & $\mathrm{~b}$ \\
\hline & Não & $0,00 \%$ & - & $\mathrm{c}^{*}$ \\
\hline & Sim & $100,00 \%$ & $\pm 6 \%$ & $\mathrm{a}$ \\
\hline Usabilidade & Médio & $9,54 \%$ & $\pm 6 \%$ & $\mathrm{~b}$ \\
\hline & Não & $0,00 \%$ & - & $\mathrm{c}^{*}$ \\
\hline
\end{tabular}

* Percentuais seguidos por pelo menos uma mesma letra na coluna de cada característica do programa não diferem entre si pelo intervalo de confiança t de Student a 5\% de probabilidade.

programa aplicável à operação a que se destina. Na confiabilidade, foi observado que $100 \%$ dos entrevistados acharam o mesmo confiável. Na avaliação do programa computacional em relação a sua característica de usabilidade, foi verificado que $100 \%$ dos entrevistados aprovaram o programa.

Para validar o programa desenvolvido, realizou-se a comparação de dados de potência demandada gerados pelo programa com resultados de trabalhos executados em condições de campo com implementos equivalentes.
Salvador et al. (2008), avaliando o desempenho de um trator e um subsolador de arrasto dotado de três hastes parabólicas com ponteiras aladas, espaçadas de 0,7 m, dispostas triangularmente e com peso de $710 \mathrm{kgf}$, em solo duro, na operação de subsolagem realizada antes e depois de diferentes sistemas de preparo periódico do solo, observaram valor médio de $23,80 \mathrm{~kW}$ para demanda de potência na barra de tração. Com a utilização do programa, observou-se o valor de 21,01 kW para a demanda de potência na barra de tração, o que está dentro do limite de aceitação da norma ASAE D497.4. 
Em trabalho realizado avaliando o desempenho de uma semeadora modelo Semeato PSM 102 em solo duro, Andreolla \& Gabriel Filho (2006) observaram que a potência exigida na barra de tração foi de $31,85 \mathrm{~kW}$ e no motor do trator de 49,81 kW para a execução dessa operação. Simulando essas condições no programa desenvolvido, obteve-se $31 \mathrm{~kW}$ para a barra de tração e $44,7 \mathrm{~kW}$ para o motor do trator, observando-se ótima correlação entre os dados reais e os simulados pelo programa.

\section{CONCLUSÕES}

O programa computacional desenvolvido tornou-se possível a execução de tarefas para cálculos de avaliação da demanda de potência de máquinas e implementos agrícolas de forma simplificada usando a internet, sem a necessidade de instalação de programas específicos.

O programa computacional desenvolvido foi avaliado obtendo boa aprovação em itens específicos como funcionalidade, objetivo, projeto visual, compatibilidade, aplicabilidade, confiabilidade e usabilidade.

Os resultados obtidos pelo programa foram validados com dados encontrados em trabalhos de pesquisa apresentando resultados bem próximos de valores práticos realizados com características práticas e teóricas semelhantes.

\section{REFERÊNCIAS}

Andreolla VRM \& Gabriel Filho A (2006) Demanda de potência de uma semeadora com dois tipos de sulcadores em áreas compactadas pelo pisoteio de animais no sistema integração lavoura-pecuária. Engenharia Agrícola, 26:768-776.

ASAE - American Society of Agricultural Engineers (2003) Agricultural machinery management data. St. Joseph, ASAE. $9 \mathrm{p}$.

Camargo WP (2005) Desenvolvimento de um ambiente web para a interação entre participantes de projetos de agricultura de precisão. Dissertação de Mestrado. Universidade de São Paulo, São Paulo. 70p.

Campos FH (2009) Desenvolvimento de um programa computacional destinado a unidade móvel de ensaio na barra de tração (UMEB) para a avaliação do desempenho de tratores. Dissertação de Mestrado. Universidade Estadual de São Paulo, São Paulo. 110p.

Gladcheff AP (2001) Um instrumento de avaliação da qualidade para software educacional de matemática. Dissertação de Mestrado. Universidade de São Paulo, São Paulo. 212p.

Heck A (2007) Desenvolvimento web para ritmo veículos. Monografia. Centro Universitário Feevale, Novo Hamburgo. $77 \mathrm{p}$.

Klaver PPC (2006) Fórum eletrônico de discussão sobre clínica fitossanitária no Brasil. Monografia. Universidade Estadual do Norte Fluminense, Campos dos Goytacazes. 56p.

Meira CAA, Mancini AL, Maximo FA, Fileto R \& Massruhá SMFS (1996) Agroinformática: qualidade e produtividade na agricultura. Cadernos de Ciência \& Tecnologia, 13:175-194.
Neto AAC, Leonel M \& Silva TPL (2009) Produção de um software para a análise da produção de mandioca, batata doce e batata no estado de São Paulo. In: Congresso Brasileiro de Mandioca, Botucatu. Anais, UNESP. p.925-930.

Pranav PK \& Pandey KP (2008) Computer simulation of ballast management for agricultural tractors. Journal of Terramechanics, 45:185-192.

Salvador N, Benez SH \& Mion RL (2008) Consumo de combustível na operação de subsolagem realizada antes e depois de diferentes sistemas de preparo periódico do solo. Engenharia Agrícola, 28:256-262.

Santos FL, Fernandes HC \& Rinaldi PCN (2006) Desenvolvimento de uma planilha eletrônica para a determinação da potência disponível na barra de tração de tratores agrícolas. Engenharia na Agricultura, 14:122-130.

Souza FM, Cunha AM, Fernandes CT \& Guerra EM (2009) Uso do GQM para avaliar documentos de utilização de framework. In: Simpósio Brasileiro de Qualidade de Software, Ouro Preto. Anais, PUC Minas. p.358-365. 